\title{
Development of Coarse-grained Molecular Dynamics Friction Simulator and Its Application to Bottlebrush Polymer
}

\author{
Shuichi UEHARA, Zhongmin LIU, Narumasa MIYAZAKI, Yusuke OOTANI, \\ Nobuki OZAWA, Momoji KUBO*
}

\begin{abstract}
Institute for Materials Research, Tohoku University, 2-1-1 Katahira, Aoba-ku, Sendai 980-8577, Japan
*e-mail:momoji@imr.tohoku.ac.jp
\end{abstract}

(Received: April 16, 2019; Accepted for publication: June 20, 2019; Online publication: July 30, 2019)

Bottlebrush polymer (BBP) absorbed on a negatively charged ceramics surface creates a low friction surface. However, the effect of the contact pressure on friction force of the surface covered by BBP is still unknown due to the difficulty in in-situ observation of the friction interface. Moreover, conventional coarse-grained molecular dynamics simulation is difficult to be directly compared to experimental data because the conventional coarse-grained model cannot consider chemical specificity of monomers. Herein, we developed a coarse-grained molecular dynamics friction simulator which can consider chemical specificity. Then, we performed friction simulation between substrates covered by BBP in water. We showed that the friction coefficient rapidly increased at contact pressure of $16 \mathrm{MPa}$, when we increased the contact pressure. We found that a sufficient coordination of water to the side chain of BBP facilitated slip between substrates under low pressure $(<16 \mathrm{MPa})$, leading to a low friction coefficient. On the other hand, under high pressure ( $>16 \mathrm{MPa}$ ), the number of side chain of BBP contacts with the BBP on the counter surface increased because water beads were squeezed out from the side chain of BBP. Therefore, the main chain of BBP was strongly stretched to the sliding direction, which induced shear between substrate and BBP. We found that the shear under high pressure caused a high friction coefficient.

Keywords: Bottle brush polymer, Friction, Martini force field, Coarse-grained Molecular dynamics, Polymer brush

\section{Introduction}

Bottlebrush polymer (BBP) is the macromolecule on which polymeric side-chains are grafted. BBP with cationic anchor absorbs on negatively charged ceramics surface and forms a low friction surface layer in aqueous environment. Therefore, BBP is potentially useful low friction material for artificial joints. Previous experimental study reported that the friction force of the surface covered by BBP increased rapidly as the contact pressure increased [1]. The rapid increase of the friction force losses the durability of artificial joints. However, the mechanism is still unknown due to the difficulty in insitu observation of the friction interface. Thus, computational simulation is required to reveal the friction mechanism. In many previous simulations, the Kremer-Grest model has been widely used to examine the frictional properties of polymer [2]. However, such a model cannot consider specific structure of polymer. Thus, it is difficult to perform simulations which can be directly compared to experimental data by using the Kremer-Grest model.

Herein, we developed a friction simulator based on Martini force field [3] which can consider chemical specificities of polymer, such as bond length and bond angle between monomers, and densities. Then, we performed friction simulations of the surface covered by BBP to reveal the effect of the pressure on friction force with a simulator developed in our group. 


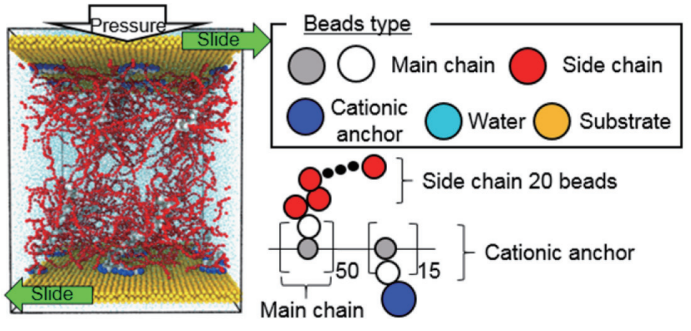

Figure 1. Siding simulation model and schematics of BBP.
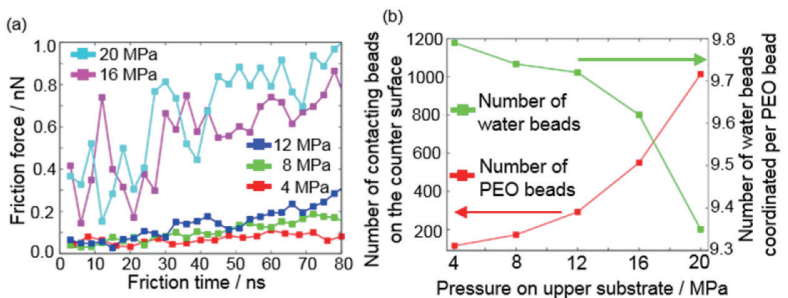

Figure 2. (a) Friction force of substrate covered by BBP. (b) Number of water beads contacting to PEO.

\section{Simulation Model}

As shown in Figure 1, BBPs with cationic anchor were placed on the substrate under water solvent. The main chain of BBP is poly methylether methacrylate. The side chain is composed of poly ethylene oxide (PEO). The cationic anchor is methacryloxyethyl trimethylammonium. In order to construct negatively charged surface, the substrate is composed of $\mathrm{SiO}_{2}$ particles of which each layer is alternatively charged positively and negatively. The outer layer of substrate is charged negatively.

\section{Results and Discussion}

To reveal the effect of the contact pressure on friction force of the surface covered by BBP, we performed friction simulation by applying a velocity of $0.25 \mathrm{~m} / \mathrm{s}$ to upper and lower substrates in opposite direction with the pressure of 4 20 MPa. Figure 2 (a) shows the variation of friction force under each pressure. We obtained the friction coefficient by averaging the friction force from 60 to $80 \mathrm{~ns}$. Under the low pressure of 4,8 and $12 \mathrm{MPa}$, friction coefficients were 0.043 , $0.040,0.040$, respectively. On the other hand, under the high pressure of $16 \mathrm{MPa}$ and $20 \mathrm{MPa}$, friction coefficients were
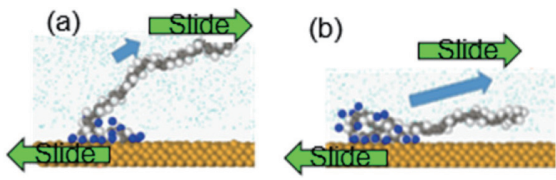

Figure 3. Dynamics of one BBP under (a) 4 and (b) $20 \mathrm{MPa}$.

$0.092,0.088$, respectively indicating a rapidly increase in the friction coefficient. This result was qualitatively consistent with experimental data showing that the friction force increased rapidly as the pressure increased [1].

To reveal the difference in the friction mechanism between the low pressure and the high pressure, we investigated the number of water beads around PEO beads (within $0.52 \mathrm{~nm}$ ) and the number of contacting PEO beads absorbed on the counter surface (Figure 2 (b)). We found that the number of water beads around the PEO beads decreased as the pressure increased, whereas the number of contacting PEO beads absorbed on the counter surface increased. Under the low pressure, the sufficient coordination of water beads around the hydrophilic PEO beads facilitated slip between substrates, leading to low friction. On the other hand, under high pressure, contact of PEO on the counter surface increased because water beads were squeezed out from PEO due to the severe pressure. Thus, the contact with PEO on the counter surface increased under high pressure. Therefore, the main chain of BBP compared to under low pressure as shown in Figure 3.

To investigate the adsorption state of BBP to the substrate during the friction, we calc ulated the moving distance of cationic anchor beads on the substrate in sliding direction. At $4 \mathrm{MPa}$ and $20 \mathrm{MPa}$, the moving distance of cationic anchor beads on the substrate was 0.35 and $2.21 \mathrm{~nm}$ respectively. The larger moving distance under high pressure indicated that stretch of the main chain induced the shear between cationic anchor and substrate. The shear caused the large force opposite to the sliding direction of substrate, due to the strong electrostatic interaction between cationic anchor and the negatively charged substrate. Thus, the shear led to a high friction coefficient under high pressure. 


\section{Conclusion}

We developed a friction simulator based on Martini force field which can consider chemical specificities and performed friction simulations of the surface covered by BBP. We found that, under low pressure, a sufficient coordination of water around the hydrophilic side chain facilitated slip between substrates, leading to a low friction coefficient. On the other hand, contact of PEO on the counter surface increased under high pressure because water beads were squeezed out from PEO due to the severe pressure. Thus, the main chain of BBP was stretched, which induced shear between cationic anchor and substrate. The shear led to a high friction coefficient.

\section{Reference}

[1] X. Liu, E. Thormann, A. Dedinaite, M. Rutland, C. Visnevskij, R. Makuska, P. M. Claesson, Soft Matter, 9, 5361 (2013). DOI:10.1039/c3sm27862j

[2] S. de Beer, M. H. Müser, Macromolecules, 47, 7666 (2014). DOI:10.1021/ma501718b

[3] S. J. Marrink, H. J. Risselada, S. Yefimov, D. P. Tieleman, A. H. de Vries, J. Phys. Chem. B, 111, 7812 (2007). PMID:17569554, DOI:10.1021/jp071097f 